Pacific Journal of Mathematics

CHARACTERS OF INDUCED REPRESENTATIONS AND 


\title{
CHARACTERS OF INDUCED REPRESENTATIONS AND WEIGHTED ORBITAL INTEGRALS
}

\author{
ReBecCA A. HerB
}

The main result of this paper is a formula relating characters of principal series representations of a reductive Lie group to weighted orbital integrals of wave packets.

1. Introduction. Let $G$ be a reductive Lie group satisfying HarishChandra's general assumptions [2]. Let $P=M A N$ be the Langlands decomposition of a cuspidal parabolic subgroup of $G$. Denote by $\varepsilon_{2}(M)$ the set of equivalence classes of irreducible unitary square integrable representations of $M$. For $\omega \in \varepsilon_{2}(M)$ and $\nu \in \mathscr{F}=a^{*}$, the real dual of the Lie algebra of $A$, let $\pi_{\omega, \nu}$ be the corresponding unitary representation of $G$ induced from $P$. Let $f$ be a wave packet corresponding to $\omega$. Then the integral of $f$ over any regular (semisimple) orbit of $G$ which can be represented by an element of $L=M A$ has been evaluated by HarishChandra in terms of the character $\Theta_{\omega, \nu}$ of $\pi_{\omega, \nu}[4]$.

Let $\gamma$ be a regular element of $G$ contained in a Cartan subgroup $H$ of $L$. Write $H=H_{K} H_{p}$ where $H_{K}$ is compact, $H_{p}$ is split, and $A \subseteq H_{p}$. Then for suitable normalizations of the $G$-invariant measure $d \dot{x}$ on $H_{p} \backslash G$ and Haar measure $d \nu$ on $\mathscr{F}$.

$$
\int_{H_{p} \backslash G} f\left(x^{-1} \gamma x\right) d \dot{x}=\varepsilon(A, H)[W(\omega)]^{-1} \int_{\mathscr{F}}\left\langle\Theta_{\omega, \nu}, f\right\rangle \Theta_{\omega, \nu}(\gamma) d \nu
$$

where $W(\omega)=\left\{s \in N_{G}(A) / L \mid s \omega=\omega\right\}$ and $\varepsilon(A, H)$ is 1 if $H_{p}=A$ and is 0 otherwise. This formula can be interpreted as giving the value of $\Theta_{\omega, \nu}$ on regular elements $\gamma$ of a fundamental Cartan subgroup of $L$ in terms of the integral of a wave packet for $\omega$ over the orbit of $\gamma$. It also gives the Fourier inversion formula for the tempered invariant distribution

$$
f \rightarrow\langle\Lambda(\gamma), f\rangle=\int_{H_{p} \backslash G} f\left(x^{-1} \gamma x\right) d \dot{x}
$$

restricted to the subspace of $\mathscr{C}(G)$, the Schwartz space of $G$, spanned by wave packets corresponding to representations induced from cuspidal parabolic subgroups $P=M A N$ with $A \subseteq H_{p}$. The complete Fourier inversion formula for $\Lambda(\gamma)$ is much more complicated. (See [5].) 
In the case that $P=G$ is cuspidal and $\omega \in \varepsilon_{2}(G)$, then $\Theta_{\omega}$ is a discrete series character of $G$, and $f$ is a matrix coefficient corresponding to $\omega$. Formula (1.1) becomes

$$
\int_{H_{p} \backslash G} f\left(x^{-1} \gamma x\right) d \dot{x}=\varepsilon(1, H)\left\langle\Theta_{\omega}, f\right\rangle \Theta_{\omega}(\gamma)
$$

Arthur has obtained the following generalization of (1.2) [1]. Let $A$ be the split component of a parabolic subgroup of $G$. Let $L$ be the centralizer in $G$ of $A$. Corresponding to $A$, Arthur defines a function $v_{A}$ on $G$ which is left $L$-invariant. Let $\gamma$ be a regular element of $G$ contained in a Cartan subgroup $H=H_{K} H_{p}$ of $L$. Let $\omega \in \varepsilon_{2}(G)$, and let $f$ be a matrix coefficient for $\omega$. Then Arthur's formula is

$$
\int_{H_{p} \backslash G} f\left(x^{-1} \gamma x\right) v_{A}(x) d \dot{x}=(-1)^{p} \varepsilon(A, H)\left\langle\Theta_{\omega}, f\right\rangle \Theta_{\omega}(\gamma)
$$

where $p$ is the dimension of $A$. This formula gives the value of the character $\Theta_{\omega}$ on the nonelliptic element $\gamma$ in terms of a weighted orbital integral of a matrix coefficient of $\omega$. It also gives the Fourier inversion formula for the tempered distribution

$$
f \rightarrow\left\langle r_{A}(\gamma), f\right\rangle=\int_{H_{p} \backslash G} f\left(x^{-1} \gamma x\right) v_{A}(x) d \dot{x}
$$

restricted to the space ${ }^{0} \mathscr{C}(G)$ of cusp forms on $G$. The distributions $r_{A}(\gamma)$ occur in the Selberg trace formula for $\Gamma \backslash G, \Gamma$ a discrete subgroup of $G$ for which $\Gamma \backslash G$ has finite volume but is not compact. As formula (1.3) shows, $r_{A}(\gamma)$ is invariant on ${ }^{0} \mathscr{C}(G)$. However, $r_{A}(\gamma)$ is not an invariant distribution on $\mathscr{C}(G)$, and the full Fourier inversion formula for $r_{A}(\gamma)$ is not known.

Authur's formula can be generalized to the setting of induced representations and wave packets. Let $P=M A N$ be a cuspidal parabolic subgroup of $G$, and let $A_{1}$ be the split component of a parabolic subgroup of $L=M A, L_{1}$ its centralizer in $G$. Let $\gamma$ be a regular element of $G$ contained in a Cartan subgroup $H=H_{K} H_{p}$ of $L_{1}$. We will define a left $L_{1}$-invariant function $v_{A_{1}}^{P}$ on $G$ with the following properties.

If $f^{\prime}$ is a wave packet coming from a cuspidal parabolic subgroup $P^{\prime}=M^{\prime} A^{\prime} N^{\prime}$ of $G$ with $\operatorname{dim} A^{\prime} \leq \operatorname{dim} A$ and $A$ not conjugate to $A^{\prime}$, then

$$
\int_{H_{p} \backslash G} f^{\prime}\left(x^{-1} \gamma x\right) v_{A_{1}}^{P}(x) d \dot{x}=0 .
$$


Now let $f$ be a wave packet corresponding to $\omega \in \varepsilon_{2}(M)$. Then

$$
\int_{H_{p} \backslash G} f\left(x^{-1} \gamma x\right) v_{A_{1}}^{P}(x) d \dot{x}=0 \quad \text { if } H_{p} \neq A_{1} .
$$

If $H_{p}=A_{1}$, let $\gamma=\gamma_{1}, \gamma_{2}, \ldots, \gamma_{k}$ be a complete set of elements of $L$ for which $\gamma_{i}=x_{l} \gamma x_{l}^{-1}$ for some $x_{l} \in G$, but $\gamma_{i}$ and $\gamma_{J}$ are not conjugate in $L$ for $1 \leq i \neq j \leq k$. Let $A_{i}=x_{l} A_{1} x_{l}^{-1}$. Then

$$
\begin{array}{rl}
\int_{H_{p} \backslash G} & f\left(x^{-1} \gamma x\right) \sum_{l=1}^{k} v_{A_{i}}^{P}\left(x_{l} x\right) d \dot{x} \\
= & (-1)^{p_{1}}[W(\omega)]^{-1} \int_{\mathscr{F}}\left\langle\Theta_{\omega, \nu}, f\right\rangle \Theta_{\omega, \nu}(\gamma) d \nu
\end{array}
$$

where $p_{1}$ is the dimension of $A_{1} \cap M$.

Formulas (1.4)-(1.6) are proved by using Arthur's formula and results of Harish-Chandra relating characters and orbital integrals on $G$ to those on $M$ and $L$. Any unexplained notation follows that of Harish-Chandra $[2,3,4]$.

2. Background material. Let $G$ be a real reductive Lie group, $g$ the Lie algebra of $G$. Let $K$ be a maximal compact subgroup of $G, \theta$ the Cartan involution of $G$ corresponding to $K$, and $B$ a real symmetric bilinear form on $\mathfrak{g}$. Assume that $(G, K, \theta, B)$ satisfy the general assumptions of Harish-Chandra in [2] and that Haar measures are normalized as in [2]. Given a $\theta$-stable Cartan subgroup $H$ of $G$, we will write $H=H_{K} H_{p}$ where $H_{K}=H \cap K$ and $H_{p}$ is a vector subgroup with Lie algebra $\mathfrak{h}_{p}$ contained in the -1 eigenspace for $\theta$. Let $G^{\prime}$ be the set of regular semisimple elements of $G, H^{\prime}=H \cap G^{\prime}$. If $J$ is any subgroup of $G$, we will write $N_{G}(J)$ and $C_{G}(J)$ for the normalizer and centralizer of $J$ in $G$, respectively, and $W(G, J)=N_{G}(J) / C_{G}(J)$.

We will first review some definitions and formulas of Harish-Chandra from $[2,3,4]$. Fix a double unitary representation $\tau$ of $K$ on a finite-dimensional Hilbert space $V$. Let $\mathscr{C}(G, \tau)$ and ${ }^{0} \mathscr{C}(G, \tau)$ denote the $\tau$-spherical functions in the spaces of $V$-valued Schwartz functions $\mathscr{C}(G, V)$ and $V$-valued cusp forms ${ }^{0} \mathscr{C}(G, V)$ respectively. Let $F_{0}$ be the operator on $V$ given by

$$
F_{0} v=\int_{K} \tau\left(k^{-1}\right) v \tau(k) d k, \quad v \in V
$$

For $f \in \mathscr{C}(G, V)$ and $x \in G$, define $\bar{f}(x)=\int_{K} f\left(k^{-1} x k\right) d k$. Then if $f \in$ $\mathscr{C}(G, \tau), \bar{f}(x)=F_{0} f(x), x \in G$. 
Fix a cuspidal parabolic subgroup $P=M A N$, that is, a parabolic subgroup of $G$ with $\varepsilon_{2}(M) \neq \varnothing$. Let $\tau_{M}$ be the restriction of $\tau$ to $K_{M}=K \cap M$. For any $f \in \mathscr{C}(G, V), m \in M$, and $a \in A$, let

$$
f^{(P)}(m a)=f_{a}^{(P)}(m)=\delta_{P}^{1 / 2}(a) \int_{N} f(\text { man }) d n
$$

where $\delta_{P}$ is the module of $P$. Then $f_{a}^{(P)} \in \mathscr{C}(M, V), f^{(P)} \in \mathscr{C}(M A, V)$, and the following relationships between $f$ and $f^{(P)}$ can be found in or easily derived from results in $[2,3,4]$.

Let $H$ be a $\theta$-stable Cartan subgroup of $L$. for $f \in \mathscr{C}(G, V)$ and $h \in H^{\prime}$,

$$
\int_{N} f\left(n^{-1} h n\right) d n=\Delta_{+}^{G}(h)^{-1} \Delta_{+}^{L}(h) f^{(P)}(h)
$$

where $\Delta_{+}^{L}$ and $\Delta_{+}^{G}$ are the functions $\Delta_{+}$on $H$, considered as a Cartan subgroup of $L$ and $G$ respectively, defined by Harish-Chandra in [2].

For $\nu \in \mathscr{F}=a^{*}$ and $m \in M$, define

$$
f_{\nu}^{(P)}(m)=\int_{A} f^{(P)}(m a) e^{-i \nu(\log a)} d a .
$$

Then because $d \nu$ is the dual measure to $d a$ on $A$ and $f^{(P)}$ is rapidly decreasing in the $A$ variable,

$$
f^{(P)}(m a)=\int_{\mathscr{F}} f_{\nu}^{(P)}(m) e^{l \nu(\log a)} d \nu .
$$

For $\omega \in \varepsilon_{2}(M)$ and $\nu \in \mathscr{F}$, let $\pi_{\omega, \nu}$ be the tempered unitary representation of $G$ induced from $\omega \otimes e^{i \nu} \otimes 1$ on $M A N$. Let $\Theta_{\omega, \nu}$ and $\Theta_{\omega}$ denote the characters of $\pi_{\omega, \nu}$ and $\omega$ considered as functions on $G^{\prime}$ and $M^{\prime}$ respectively. For $f \in \mathscr{C}(G, \tau), g \in \mathscr{C}\left(M, \tau_{M}\right)$, define

$$
\left\langle\Theta_{\omega, \nu}, f\right\rangle=\int_{G} f(x) \overline{\Theta_{\omega, \nu}(x)} d x \quad \text { and } \quad\left\langle\Theta_{\omega}, g\right\rangle=\int_{M} g(m) \overline{\Theta_{\omega}(m)} d m .
$$

Then, for $f \in \mathscr{C}(G, \tau), \nu \in \mathscr{F}, f_{\nu}^{(P)} \in \mathscr{C}\left(M, \tau_{M}\right)$ and

$$
\left\langle\Theta_{\omega, \nu}, f\right\rangle=F_{0}\left\langle\Theta_{\omega}, f_{\nu}^{(P)}\right\rangle \text {. }
$$

For $\omega \in \varepsilon_{2}(M)$, let $L(\omega)={ }^{0} \mathscr{C}\left(M, \tau_{M}\right) \cap \mathfrak{h}_{\omega} \otimes V$ where $\mathfrak{h}_{\omega}$ is the closed subspace of $L^{2}(M)$ spanned by matrix coefficients for $\omega$. For $\psi \in L(\omega), \alpha \in C_{c}^{\infty}(\mathscr{F})$, and $x \in G$, define

$$
\varphi_{\alpha}(x)=\int_{\mathscr{F}} \alpha(\nu) E(P: \psi: \nu: x) \mu(\omega: \nu) d \nu
$$


where $E(P: \psi: \nu)$ is the Eisenstein integral defined in [2], and $\mu(\omega: \nu)$ is the Plancherel factor corresponding to $\pi_{\omega, \nu}$. Then $\varphi_{\alpha} \in \mathscr{C}(G, \tau)$ is called a wave packet for $\omega \in \varepsilon_{2}(M)$, and for $\nu \in \mathscr{F},\left(\varphi_{\alpha}\right)_{\nu}^{(P)}$ belongs to $\sum_{s \in W(G, A)} L(s \omega)$ and is supported on a compact subset of $\mathscr{F}$.

We now turn to Arthur's results. Let $A$ be a special vector subgroup of $G$, that is, the split component of a parabolic subgroup of $G$. Write $\mathscr{P}(A)$ for the (finite) set of all parabolic subgroups of $G$ having $A$ as split component. For $P \in \mathscr{P}(A)$ let $\Phi_{P}$ denote the set of simple roots of $(P, A)$. We identify $a$, the Lie algebra of $A$, and its dual via the bilinear form $B$. A set $\mathscr{Y}=\left\{Y_{P} \mid P \in \mathscr{P}(A)\right\}$ of points in $\mathfrak{a}$ is called $A$-orthogonal if for any pair of adjacent parabolic subgroups $P, P^{\prime} \in \mathscr{P}(A), Y_{P}-Y_{P^{\prime}}=r \alpha, r \in \mathbf{R}$, where $\alpha$ is the unique element of $\Phi_{P}$ with $-\alpha \in \Phi_{P^{\prime}}$. Let

$$
\mathfrak{a}^{0}=\{H \in \mathfrak{a} \mid\langle\alpha, H\rangle=0 \text { for every root } \alpha \text { of }(g, \mathfrak{a})\},
$$

$\mathfrak{a}^{1}$ its orthogonal complement in $A$. Let $p$ be the dimension of $\mathfrak{a}^{1}$, and let $c_{A}=|\operatorname{det} C|^{1 / 2}$ where $C$ is the Cartan matrix for the roots of $(\mathfrak{g}, \mathfrak{a})$. For any $P=M A N \in \mathscr{P}(A)$ and $x \in G$, write

$$
x=m(x) \exp \left(H_{P}(x)\right) n(x) k(x)
$$

where $m(x) \in M, n(x) \in N, k(x) \in K$, and $H_{P}(x) \in a$. For any $A$-orthogonal set $\mathscr{Y}$ and $\lambda \in \mathfrak{a}_{\mathbf{C}}^{1}$, define

$$
v(x: \mathscr{Y})=c_{A}(p !)^{-1} \sum_{P \in \mathscr{P}(A)} \frac{\left\langle\lambda, Y_{P}-H_{P}(x)\right\rangle^{p}}{\prod_{\alpha \in \Phi_{P}}\langle\lambda, \alpha\rangle} .
$$

Then $v(x: \mathscr{Y})$ is independent of $\lambda$ and is left-invariant under $L=C_{G}(A)$. It is also clearly right $K$-invariant. If $v_{A}(x)=v(x: \mathscr{Y})$ for any $A$-orthogonal set $\mathscr{Y}$, then (1.3) is valid.

3. The distributions. Fix a cuspidal parabolic subgroup $P=M A N$ of $G$. Let $A_{1}^{M}$ be a special vector subgroup of $M, A_{1}=A_{1}^{M} A$. Let $\mathscr{Y}_{1}$ be an $A_{1}^{M}$-orthogonal set, and let $v_{1}^{M}(m)=v\left(m: \mathscr{Y}_{1}\right), m \in M$, be the function on $M$ defined as in (2.7) with respect to $A_{1}^{M}$ and $\mathscr{Y}_{1}$. Extend $v_{1}^{M}$ to a function $v_{1}$ on $G$ by setting

$$
\begin{aligned}
& v_{1}(\text { mank })=[W(G, A)]^{-1} v_{1}^{M}(m), \\
& \quad m \in M, a \in A, n \in N, k \in K .
\end{aligned}
$$

This extension is well defined since $v_{1}^{M}$ is right $K_{M}$-invariant. Since $v_{1}^{M}$ if left-invariant under $L_{1}^{M}=C_{M}\left(A_{1}^{M}\right), v_{1}$ is left-invariant under $L_{1}=L_{1}^{M} A$ $=C_{G}\left(A_{1}\right)$. 
Let $H$ be a $\theta$-stable Cartan subgroup of $G$ with $A_{1} \subseteq H_{p}$. Write $J=H \cap M$. Let $h \in H^{\prime}$. For $f \in C_{c}^{\infty}(G, V)$, define

$$
\left\langle r_{1}(h), f\right\rangle=\int_{H_{p} \backslash G} f\left(x^{-1} h x\right) v_{1}(x) d \dot{x} .
$$

LEMMA 3.3. For any $h \in H^{\prime}$ the distribution $r_{1}(h)$ is tempered. For any $f \in \mathscr{C}(G, V), \int_{H_{p} \backslash G} f\left(x^{-1} h x\right) v_{1}(x) d \dot{x}$ is absolutely convergent and

$$
\begin{aligned}
& \left\langle r_{1}(h), f\right\rangle=\int_{H_{p} \backslash G} f\left(x^{-1} h x\right) v_{1}(x) d \dot{x} \\
& \quad=[W(G, A)]^{-1} \Delta_{+}^{G}(h)^{-1} \Delta_{+}^{L}(h) \int_{J_{p} \backslash M} \bar{f}^{(P)}\left(m^{-1} h m\right) v_{1}^{M}(m) d \dot{m} .
\end{aligned}
$$

Proof. Let $f \in \mathscr{C}(G, V)$. Write $h=j a$ where $j \in J^{\prime}, a \in A$. Then using (2.2) and (3.1),

$$
\begin{aligned}
\int_{H_{p} \backslash G} \mid & f\left(x^{-1} h x\right) v_{1}(x) \mid d \dot{x} \\
& =[W(G, A)]^{-1} \int_{J_{p} \backslash M}\left|v_{1}^{M}(m)\right| \int_{N K}\left|f\left(k^{-1} n^{-1} m^{-1} h m n k\right)\right| d n d k d \dot{m} \\
& =[W(G, A)]^{-1} \Delta_{+}^{G}(h)^{-1} \Delta_{+}^{L}(h) \int_{J_{p} \backslash M}\left|v_{1}^{M}(m) \bar{f}_{a}^{(P)}\left(m^{-1} j m\right)\right| d \dot{m}
\end{aligned}
$$

since $\Delta_{+}^{G}$ and $\Delta_{+}^{L}$ are invariant under conjugation by $M$. The lemma now follows since for any $a \in A, f \rightarrow \bar{f}_{a}^{(P)}$ is a continuous map from $\mathscr{C}(G, V)$ to $\mathscr{C}(M, V)$ [2]. Further, for $g \in \mathscr{C}(M, V), j \in J^{\prime}$,

$$
\int_{J_{p} \backslash M} g\left(m^{-1} j m\right) v_{1}^{M}(m) d \dot{m}
$$

is absolutely convergent and defines a tempered distribution [1].

COROLlaRY 3.4. Let $A^{\prime}$ be a special vector subgroup of $G$ with $\operatorname{dim} A^{\prime}$ $\leq \operatorname{dim} A$. Let $P^{\prime}=M^{\prime} A^{\prime} N^{\prime} \in \mathscr{P}\left(A^{\prime}\right), \omega^{\prime} \in \varepsilon_{2}\left(M^{\prime}\right)$. Let $f$ be a wave packet defined as in (2.6) with respect to $\omega^{\prime}$ and $P^{\prime}$. Then $\left\langle r_{1}(h), f\right\rangle=0$ unless $A^{\prime}$ is conjugate to $A$ under $K$.

Proof. In this case $\bar{f}^{(P)}=0$ [4]. Thus the result follows from (3.3). 
LEMMA 3.5 Suppose that $f=\varphi_{\alpha}$ is a wave packet associated to $\omega \in$ $\varepsilon_{2}(M)$. Let $h \in H^{\prime}$. Then

$$
\begin{aligned}
\left\langle r_{1}(h), f\right\rangle= & {[W(G, A)]^{-1}(-1)^{p_{1}} \varepsilon\left(A_{1}, H\right)[W(\omega)]^{-1} \Delta_{+}^{G}(h)^{-1} \Delta_{+}^{L}(h) } \\
& \cdot \int_{\mathscr{F}}\left\langle\Theta_{\omega, \nu}, f\right\rangle \sum_{s \in W(G, A)}\left(\Theta_{s \omega} \otimes e^{i s \nu}\right)(h) d \nu
\end{aligned}
$$

where $p_{1}=\operatorname{dim} A_{1}^{M}$.

Proof. Using (3.3) and (2.4),

$$
\begin{aligned}
\left\langle r_{1}(h), f\right\rangle= & {[W(G, A)]^{-1} \Delta_{+}^{G}(h)^{-1} \Delta_{+}^{L}(h) F_{0} } \\
& \cdot \int_{J_{p} \backslash M} v_{1}^{M}(m) \int_{\mathscr{F}} e^{i \nu(\log a)} f_{\nu}^{(P)}\left(m^{-1} j m\right) d \nu d \dot{m} .
\end{aligned}
$$

Since $f_{\nu}^{(P)} \in \mathscr{C}(M, V)$ and is supported on a compact subset of $\mathscr{F}$, we can interchange the order of integration. Let $W=W(G, A)$, and write $f_{\nu}^{(P)}=$ $\sum_{s \in W / W(\omega)} g_{s}$ where $g_{s} \in L(s \omega)$. Then, using (1.3),

$$
\int_{J_{p} \backslash M} g_{s}\left(m^{-1} j m\right) v_{1}^{M}(m) d \dot{m}=(-1)^{p_{1}} \varepsilon\left(A_{1}^{M}, J\right)\left\langle\Theta_{s \omega}, g_{s}\right\rangle \Theta_{s \omega}(j) .
$$

But $\varepsilon\left(A_{1}^{M}, J\right)=\varepsilon\left(A_{1}, H\right)$, and $\left\langle\Theta_{\mathrm{s} \omega}, g_{s^{\prime}}\right\rangle=0$ if $s \omega \neq s^{\prime} \omega$. Thus using (2.5),

$$
\begin{aligned}
F_{0} \int_{J_{p} \backslash M} f_{\nu}^{(P)}\left(m^{-1} j m\right) v_{1}^{M}(m) d \dot{m} \\
\quad=(-1)^{p_{1}} \varepsilon\left(A_{1}, H\right) \sum_{s \in W / W(\omega)} F_{0}\left\langle\Theta_{s \omega}, f_{\nu}^{(P)}\right\rangle \Theta_{s \omega}(j) \\
\quad=(-1)^{p_{1}} \varepsilon\left(A_{1}, H\right)[W(\omega)]^{-1} \sum_{s \in W}\left\langle\Theta_{s \omega, \nu}, f\right\rangle \Theta_{s \omega}(j) .
\end{aligned}
$$

Now for each $s \in W$,

$$
\int_{\mathscr{F}} e^{i \nu(\log a)} \Theta_{s \omega}(j)\left\langle\Theta_{s \omega, \nu}, f\right\rangle d \nu=\int_{\mathscr{F}} e^{i s \nu(\log a)} \Theta_{s \omega}(j)\left\langle\Theta_{\omega, \nu}, f\right\rangle d \nu
$$

since $\Theta_{s \omega, s \nu}=\Theta_{\omega, \nu}$.

Now suppose that $H$ is a Cartan subgroup of $G$ with $H_{p}=A_{1}$, and fix $h \in H^{\prime}$. Let $h_{i}=x_{i} h x_{i}^{-1}, 1 \leq i \leq k$, be defined as in (1.6). Then using 
results from $[6,7]$, for $\omega \in \varepsilon_{2}(M)$ and $\nu \in \mathscr{F}$,

$$
\Theta_{\omega, \nu}(h)=\sum_{i=1}^{k} \Delta_{+}^{G}\left(h_{i}\right)^{-1} \Delta_{+}^{L}\left(h_{i}\right)\left(\Theta_{\omega} \otimes e^{i \nu}\right)\left(h_{l}\right) .
$$

Fix $1 \leq i \leq k$. Let $A_{i}=x_{i} A_{1} x_{l}^{-1}, A_{i}^{M}=A_{i} \cap M$. Let $L_{i}=C_{G}\left(A_{i}\right)$. Then $A_{i}^{M}$ is a special vector subgroup of $M$. Let $\mathscr{Y}_{i}$ be any $A_{i}^{M}$-orthogonal set, and define $v_{l}$ on $G$ as in (3.1) starting from $v_{l}^{M}$. Then $v_{i}$ is left $L_{i}$-invariant so that $x \rightarrow v_{t}\left(x_{i} x\right)$ is left $L_{1}$-invariant. For $x \in G$ define

$$
\langle r(h), f\rangle=\int_{H_{p} \backslash G} f\left(x^{-1} h x\right) \sum_{i=1}^{k} v_{i}\left(x_{i} x\right) d \dot{x} .
$$

THEOREM 3.8. Let $H$ be a Cartan subgroup of $G$ with $H_{p}=A_{1}, h \in H^{\prime}$. Then $r(h)$ is a tempered distribution, and for $f$ a wave packet corresponding to $\omega \in \varepsilon_{2}(M)$,

$$
\langle r(h), f\rangle=(-1)^{p_{1}}[W(\omega)]^{-1} \int_{\mathscr{F}}\left\langle\Theta_{\omega, \nu}, f\right\rangle \Theta_{\omega, \nu}(h) d \nu .
$$

Proof. Define $x_{1}, \ldots, x_{k}$ and $h_{1}, \ldots, h_{k}$ as in (3.6). Then for $1 \leq i \leq k$, $H_{i}=x_{i} H x_{i}^{-1}$ is a Cartan subgroup of $G$ with $A_{i}=\left(H_{t}\right)_{p}$ so that using (3.3),

$$
\begin{aligned}
\langle r(h), f\rangle & =\sum_{i=1}^{k} \int_{H_{p} \backslash G} f\left(x^{-1} h x\right) v_{i}\left(x_{i} x\right) d \dot{x} \\
& =\sum_{i=1}^{k} \int_{\left(H_{i}\right)_{p} \backslash G} f\left(x^{-1} h_{l} x\right) v_{i}(x) d \dot{x} \\
& =(-1)^{p_{1}} \varepsilon\left(A_{1}, H\right)[W(\omega)]^{-1} \int_{\mathscr{F}}\left\langle\Theta_{\omega, \nu}, f\right\rangle \varphi(\omega, \nu, h) d \nu
\end{aligned}
$$

where

$$
\begin{aligned}
\varphi(\omega, \nu, h) & =[W]^{-1} \sum_{s \in W} \sum_{i=1}^{k} \Delta_{+}^{G}\left(h_{i}\right)^{-1} \Delta_{+}^{L}\left(h_{i}\right) \Theta_{s \omega} \otimes e^{i s \nu}\left(h_{i}\right) \\
& =[W]^{-1} \sum_{s \in W} \Theta_{s \omega, s \nu}(h)=\Theta_{\omega, \nu}(h) .
\end{aligned}
$$




\section{REFERENCES}

[1] J. Arthur, The characters of discrete series as orbital integrals, Inv. Math., 32 (1976), 205-261.

[2] Harish-Chandra, Harmonic analysis on real reductive groups, I, J. Funct. Anal., 19 (1975), 104-204.

[3] __ Harmonic analysis on real reductive groups, II, Inv. Math., 36 (1976), 1-55.

[4] Harmonic analysis on real reductive groups, III, Ann. of Math., 104 (1976), 117-201.

[5] R. Herb, Discrete series characters and Fourier inversion on semisimple real Lie groups, Trans. Amer. Math. Soc., 277 (1983), 241-261.

[6] W. Schmid, On the characters of the discrete series, Inv. Math., 30 (1975), 47-144.

[7] J. Wolf, Unitary representations on partially holomorphic cohomology spaces, Mem. Amer. Math. Soc., 138 (1974).

Received March 20, 1981. Supported in part by National Science Foundation Grant MCS 77-18723 A04.

UNIVERSITY OF MARYLAND

College Park, MD 20742 



\section{PACIFIC JOURNAL OF MATHEMATICS EDITORS}

\author{
DONALD BABBITT (Managing Editor) \\ University of California \\ Los Angeles, CA 90024 \\ J. DugundJI \\ University of Southern California \\ Los Angeles, CA 90089-1113 \\ R. FINN \\ Stanford University \\ Stanford, CA 94305 \\ HERMANN FLASChKa \\ University of Arizona \\ Tucson, AZ 85721
}

C. C. MOORE

University of California

Berkeley, CA 94720

Arthur Ogus

University of California

Berkeley, CA 94720

Hugo RossI

University of Utah

Salt Lake City, UT 84112

H. SAMELSON

Stanford University

Stanford, CA 94305

ASSOCIATE EDITORS
R. ARENS
E. F. BECKENBACH
B. H. NeUmanN
F. WOLF
K. YosHIDA (1906-1982)

\section{SUPPORTING INSTITUTIONS}
UNIVERSITY OF ARIZONA
UNIVERSITY OF BRITISH COLUMBIA
CALIFORNIA INSTITUTE OF TECHNOLOGY
UNIVERSITY OF CALIFORNIA
MONTANA STATE UNIVERSITY
UNIVERSITY OF NEVADA, RENO
NEW MEXICO STATE UNIVERSITY
OREGON STATE UNIVERSITY

\author{
UNIVERSITY OF OREGON \\ UNIVERSITY OF SOUTHERN CALIFORNIA \\ STANFORD UNIVERSITY \\ UNIVERSITY OF HAWAII \\ UNIVERSITY OF TOKYO \\ UNIVERSITY OF UTAH \\ WASHINGTON STATE UNIVERSITY \\ UNIVERSITY OF WASHINGTON
}

The Supporting Institutions listed above contribute to the cost of publication of this Journal, but they are not owners or publishers and have no responsibility for its content or policies.

Mathematical papers intended for publication in the Pacific Journal of Mathematics should be in typed form or offset-reproduced (not dittoed), double spaced with large margins. Please do not use built up fractions in the text of the manuscript. However, you may use them in the displayed equations. Underline Greek letters in red, German in green, and script in blue. The first paragraph must be capable of being used separately as a synopsis of the entire paper. In particular it should contain no bibliographic references. Please propose a heading for the odd numbered pages of less than 35 characters. Manuscripts, in triplicate, may be sent to any one of the editors. Please classify according to the scheme of Math. Reviews, Index to Vol. 39. Supply name and address of author to whom proofs should be sent. All other communications should be addressed to the managing editor, or Elaine Barth, University of California, Los Angeles, California 90024.

There are page-charges associated with articles appearing in the Pacific Journal of Mathematics. These charges are expected to be paid by the author's University, Government Agency or Company. If the author or authors do not have access to such Institutional support these charges are waived. Single authors will receive 50 free reprints; joint authors will receive a total of 100 free reprints. Additional copies may be obtained at cost in multiples of 50 .

The Pacific Journal of Mathematics is issued monthly as of January 1966. Regular subscription rate: $\$ 190.00$ a year (5 Vols., 10 issues). Special rate: $\$ 66.00$ a year to individual members of supporting institutions.

Subscriptions, orders for numbers issued in the last three calendar years, and changes of address should be sent to Pacific Journal of Mathematics, P.O. Box 969, Carmel Valley, CA 93924, U.S.A. Old back numbers obtainable from Kraus Periodicals Co., Route 100, Millwood, NY 10546.

The Pacific Journal of Mathematics at P.O. Box 969, Carmel Valley, CA 93924 (ISSN 0030-8730) publishes 5 volumes per year. Application to mail at Second-class postage rates is pending at Carmel Valley, California, and additional mailing offices. Postmaster: Send address changes to Pacific Journal of Mathematics, P.O. Box 969, Carmel Valley, CA 93924.

PUBLISHED BY PACIFIC JOURNAL OF MATHEMATICS, A NON-PROFIT CORPORATION

Copyright $\odot 1984$ by Pacific Journal of Mathematics 


\section{Pacific Journal of Mathematics}

Vol. 114, No. $2 \quad$ June, 1984

William Allen Adkins, A Harnack estimate for real normal surface

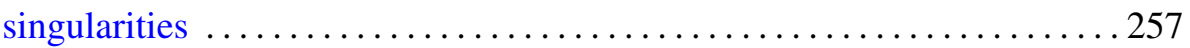

George E. Andrews, Multiple series Rogers-Ramanujan type identities . . . . 267

Didier Arnal, $*$ products and representations of nilpotent groups . . . . . . 285

David Cox and Walter Raymond Parry, Representations associated with

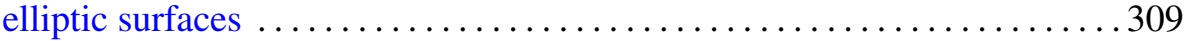

Joanne Marie Dombrowski, Tridiagonal matrix representations of cyclic

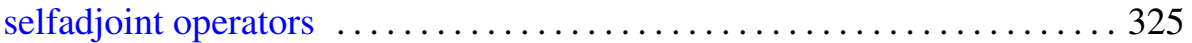

Ronald Dotzel, An Artin relation $(\bmod 2)$ for finite group actions on

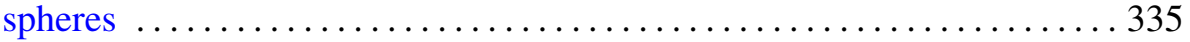

Leo Egghe, Convergence of adapted sequences of Pettis-integrable

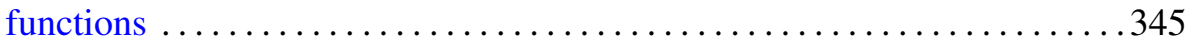

Rebecca A. Herb, Characters of induced representations and weighted

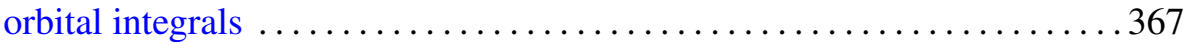

Steven M. Kahn, Cobordism obstructions to fibering manifolds over

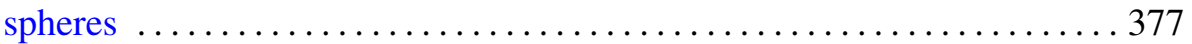

Robert D. Little, Projective space as a branched covering of the sphere with orientable branch set

Claude Schochet, Topological methods for $C^{*}$-algebras. III. Axiomatic

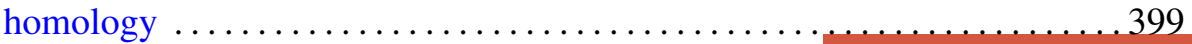

Claude Schochet, Topological methods for $C^{*}$-algebras. IV. $\bmod p$ homology

James M. Stormes, On the $K O$-orientability of complex projective varieties

Josephine Anne Ward, Characterization of homogeneous spaces and their norms 